\title{
Identification of primary genes in glomeruli compartment of immunoglobulin A nephropathy by bioinformatic analysis
}

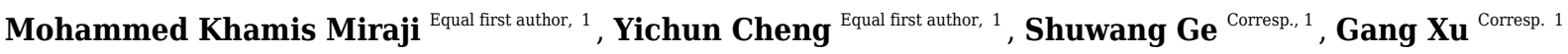 \\ ${ }^{1}$ Department of Nephrology, Tongji Hospital affiliated to Tongji Medical College, Huazhong University of Science and Technology, Wuhan, Hubei, China \\ Corresponding Authors: Shuwang Ge, Gang Xu \\ Email address: geshuwang@tjh.tjmu.edu.cn, xugang@tjh.tjmu.edu.cn
}

The current study is aimed to explore the specific genes which are responsible for the manifestation of Immunoglobulin A nephropathy (IgAN). Gene expression profiles GSE37460, GSE93798 and GSE104948 were analyzed using biological informatics methods to identify differentially expressed genes (DEGs) in IgAN glomeruli samples which were then compared to normal control samples. Subsequently, the DEGs were overlapped to explore genes with significant expression in at least two profiles. Finally, the enrichment analysis was conducted and the protein-protein interaction (PPI) network was constructed for the overlapping DEGs. A total of 28 genes were up-regulated and whereas 10 genes were down-regulated. The up-regulated genes including CD44 and FN1 were chiefly involved in extracellular matrix receptors interaction pathway. In addition, CX3CR1 and CCL4 were associated with chemokine signaling pathway. ITGB2, PTPRC, FN1, and FCER1G were hub genes with a high degree of interaction in the PPI network. Therefore, this study identified many significant genes associated with extracellular matrix expansion and inflammatory mechanism which may be the novel biomarker and target candidates in IgAN. 
$6 \quad \dagger$ Equal contributors

7 Corresponding Authors:

8 Shuwang Ge, Gang Xu

9 Email: geshuwang@,tjh.tjmu.edu.cn xugang@tjh.tjmu.edu.cn nephropathy by bioinformatic analysis

Mohammed Khamis Miraji ${ }^{\dagger}$, Yichun Cheng ${ }^{\dagger}$, Shuwang Ge ${ }^{\ddagger}$, Gang Xu $\mathrm{Xu}^{\ddagger}$

Department of Nephrology, Tongji Hospital affiliated to Tongji medical college, Huazhong University of Science and Technology, Wuhan, 430030, P.R. China.

\section{ABSTRACT}

The current study is aimed to explore the specific genes which are responsible for the manifestation of Immunoglobulin A nephropathy (IgAN). Gene expression profiles GSE37460, GSE93798 and GSE104948 were analyzed using biological informatics methods to identify differentially expressed genes (DEGs) in IgAN glomeruli samples which were then compared to normal control samples. Subsequently, the DEGs were overlapped to explore genes with significant expression in at least two profiles. Finally, the enrichment analysis was conducted and the protein-protein interaction (PPI) network was constructed for the overlapping DEGs. A total of 28 genes were up-regulated and whereas 10 genes were down-regulated. The upregulated genes including CD44 and FN1 were chiefly involved in extracellular matrix receptors interaction pathway. In addition, CX3CR1 and CCL4 were associated with chemokine signaling pathway. ITGB2, PTPRC, FN1, and FCER1G were hub genes with a high degree of interaction in the PPI network. Therefore, this study identified many significant genes associated with 
24

25

26

27

28

extracellular matrix expansion and inflammatory mechanism which may be the novel biomarker and target candidates in IgAN.

\section{INTRODUCTION}

Immunoglobulin A nephropathy (IgAN) is the most common type of biopsy-proven primary glomerulonephritis and highly prevails in Asia, especially in China where it accounts for $58.2 \%$ of the glomerulonephritis cases (Donadio \& Grande 2002). The outcome of IgAN is highly variable, $15 \sim 40 \%$ of the patients with IgAN progressively develop to end-stage renal disease (ESRD) within 20 years after diagnosis (Barbour et al. 2013). Therefore, IgAN has been a health concern throughout the world.

Pathogenic steps of the IgAN include deposition of IgA immune complexes, mesangial cells proliferation, and over-production of extracellular matrix components and infiltration of the inflammatory cells in the kidney tissues. Although these pathogenic steps of IgAN have been studied for decades (Wyatt \& Julian 2013), their mechanism is still unclear.

Gene microarray analysis is a great technique to detect the expression of thousands of genes and become an important technology for identifying genes and biological pathways that associate with various diseases. This approach is useful for identifying potential diagnostic, prognostic and therapeutic biomarker and has been applied for gene expression profiling in human IgAN (Cox et al. 2010; Liu et al. 2017). In addition, the protein-protein interaction (PPI) network was used to explore the function of proteins and disclose the rules of cellular activities including growth, development, metabolism, differentiation, and apoptosis (Szklarczyk et al. 2011). Recognition of protein interaction in a genetic study is essential in understanding the cellular control mechanism of the proteins.

In order to reveal crucial candidate genes contributing to IgAN, we conducted a series of microarray analysis of three microarray datasets which were obtained from the Gene Expression Omnibus (GEO) database. The genes with different expression (DEG) between IgAN patients 
49

and normal subjects were identified, and the overlapping DEGs were selected to perform enrichment analysis and construct a protein-protein interaction (PPI) network.

\section{METHODS}

\section{Data resources}

We searched the GEO database (http://www.ncbi.nlm.nih.gov/geo/) with keywords "IgA nephropathy" and "Gene expression profile". By January 24, 2019, a total of 22 datasets were considered. These microarray datasets were selected based on the criteria that the samples must be human glomeruli tissue and contain IgA nephropathy and healthy subjects. Finally, 3 datasets were retained for subsequent analysis (Table 1). The process of data collection and selection was provided in Fig. S1.

\section{Data processing and differential genes analysis}

The raw data was collected in the format of a CEL file and an annotation file. The data was initially preprocessed for background correction and quantile normalization using the Robust Multi-array Average (RMA) algorithm in the Affy package (Kauffmann et al. 2009). Quality control was performed by using MetaQC package (Wang et al. 2012), which provides four quantitative quality control indexes, including internal quality control (IQC), external quality control (EQC), accuracy quality control (AQCg and AQCp) and consistent quality control (CQCg and CQCp). When multiple probes matched to the same gene, we adopted the "IQR" method to select a probe with the largest interquartile range of gene expression values among all matched probes to represent the gene.

\section{Identification of the overlap DEGs between 3 microarray datasets}

The processed data was used to run the linear Model for Microarray (LIMMA) package in R (Ritchie et al. 2015). The model was applied to identify differentially expressed genes between IgAN patients and healthy subjects in each dataset. The multiple testing correction was carried out 
73 to control the false discovery rate (FDR) with the application of the Benjamini-Hochberg

74 procedure (Benjamini \& Hochberg 1995). The genes with the corrected $\mathrm{p}$ value $<0.05$ and

$75\left|\log _{2} \mathrm{FC}\right|>1$ were considered as DEGs. Then we examined the overlaps of the top 100 ranked genes

76 across the three series. Genes with significantly differential expression in at least 2 datasets were

77 selected as common genes. In order to evaluate the reliability of the above DEGs, we also

78 performed a meta-analysis by the Fisher method and the maximum P-value method in MetaDE

79 package (Wang et al. 2012).

\section{Enrichment analysis of the overlapped DEGs}

82 The function and pathway enrichment analysis of the overlapped differentially expressed genes

83 were carried out using the online web resource Database for Annotation, Visualization and Integration Discovery (DAVID, http://david.abcc.ncifcrf.gov). DAVID was used to conduct Gene Ontology (GO) and Kyoto Encyclopedia of Genes and Genomes (KEGG) pathway analysis in upregulated and down-regulated overlapping DEGs independently. The $p<0.01$ and Gene count (number of enriched genes in a specific function or a pathway) $>2$ were considered the significant threshold values for the GO terms and pathway terms enrichment in the DEGs.

\section{Construction of the PPI network}

To understand the interactions of the overlapped DEGs at the molecular level, we constructed the PPI network using the Search Tool for the Retrieval of Interacting Genes database (STRING, http://string.embl.de/) (Szklarczyk et al. 2011). The protein pairs with a confidence score $>0.6$ were considered to be significant. PPI network was visualized using Cytoscape software (http://cytoscape.org/) (Smoot et al. 2011).

\section{RESULTS}


97

98

99

100

101

102

103

104

105

106

107

108

109

110

111

112

113

114

115

116

117

118

119

120

121

122

\section{DEGs and overlap DEGs identified in 3 microarray datasets}

After the raw data of the three datasets were normalized, quality control was further performed for the datasets (Table S1). All three datasets were included for the subsequent analysis. A total of 217, 5399 and 564 genes from GSE37450, GSE93798 and GSE104948 dataset were differentially expressed between IgA nephropathy and health controls respectively. 28 upregulated and 10 down-regulated DEGs were identified in at least two datasets based on top 100 DEGs in the overlapping analysis (Fig. 1). Comparatively, FN1 gene and ALB gene were commonly expressed in all three datasets for up-regulation and down-regulation profiles respectively (Table 2).

\section{Functional and pathway enrichment of overlapping DEGs}

The DEGs were annotated in the GO database and assigned to three categories, including biological processes, molecular functions and cellular components (Fig 2). Biological process enrichment analysis indicated that these up-regulated DEGs were mainly involved in cell adhesion, leukocyte migration, and inflammatory response. Down-regulated DEGs were mainly involved in positive regulation of transcription from RNA polymerase II promoter, skeletal muscle cell differentiation and negative regulation of transcription from RNA polymerase II promoter. Cellular components analysis showed that up-regulated DEGs were mainly involved in the extracellular region, collagen trimer and extracellular space. Down-regulated DEGs were mainly involved in a nucleus. The molecular function analysis showed that up-regulated DEGs were mainly involved in protein binding receptor binding and collagen binding. Down-regulated DEGs were mainly involved in sequence-specific DNA binding, transcriptional activator activity, and DNA binding.

In addition, the up-regulated DEGs were significantly enriched in 12 pathways such as NF-kappa B signaling pathway including LYN, LY96, CCL4, CD14; ECM-receptor interaction pathway including CD44, COL6A3, COL1A2, FN1 and Amoebiasis pathway including COL1A2, ITGB2, CD14, FN1 (Table 3). No significant pathways were involved with down-regulated DEGs. 
123

124

125

126

127

128

129

130

131

132

133

134

135

136

137

138

139

140

141

142

143

144

145

146

147

\section{PPI network of overlapping DEGs}

The resultant network contained 29 nodes and 60 edges (Fig 3). Furthermore, the overlapped DEGs such as ITGB2, PTPRC, FN1, and FCER1G in the PPI network were identified as hub genes.

\section{DISCUSSION}

IgA nephropathy is the leading form of glomerulonephritis worldwide (Wyatt \& Julian 2013). Many studies have been conducted to explore the pathogenesis of IgAN (Robert et al. 2015; Suzuki et al. 2011), however, the mechanism underlying IgAN progression has not been fully elucidated. In this paper, we identified candidate genes critical to IgAN by combining three datasets from GEO database and presented the potential pathway that may play an important role in IgAN.

Our bioinformatic analysis demonstrated that FN1 is overexpressed in IgAN glomeruli. The fibronectin is an essential element of the extracellular matrix. In pathological conditions, fibronectin could act as a seed for the deposition of ECM proteins around somatic cells, leading to sclerosis or fibrosis of tissue (Brotchie \& Wakefield 1990). Besides, some animal experiments have shown that fibronectin is associated with the progression of kidney disease (Kubosawa, $\mathrm{H}$. \& Kondo 1998; Shui et al. 2006). In patients with glomerulonephritis, increased plasma and urinary fibronectin levels were observed (Altunkova et al. 1993; Idasiak-Piechocka et al. 2010). The previous study also revealed that patients with IgAN have high circulating complexes containing Ag antibodies and Fibronectin (Cederholm et al., 1988), as it is in the preferred immune complex mechanism of primary IgA nephropathy. Therefore, the expression of FN1 gene may also influence the progression of $\operatorname{IgAN}$, and thereby proper regulation of FN1 expression can effectively prevent IgAN.

Chemokines and chemokine receptors are potential in the homing and recruitment of specific immune cells. The existence of chemokine receptors expressing cells has been reported in patients with different types of nephropathies (Zhuang et al. 2017). Our results demonstrate significant over-expression of chemokine (CX3-C motif) receptor 1 (CX3CR1) and chemokine (C-C motif) 
148 ligand 4 (CCL4) which also participate in the chemokine signal pathway. CX3CR1 is a 149 transmembrane protein which is involved in the adhesion and migration of leukocytes and 150 enhances the infiltration of cytotoxic lymphocytes (Nishimura et al. 2002), CX3CL1/CX3CR1 151 axis can initiate a cascade via several signaling pathways in the kidney, including ROS/MAPKS, 152 Raf/MEK1/2-ERK1/2-Akt/PI3K and nuclear factor of kappa (NF-kB) light polypeptide gene 153 enhancer in B cells (Zhuang et al. 2017). Also, it is reported that the CX3CL1/CX3CR1 axis can 154 up-regulate mesangial cell expansion directly via Reactive Oxygen Species (ROS) and Mitogen155 Activated Protein Kinase (MAPK) in diabetic nephropathy (Park et al. 2012). The amount of 156 glomerular and urinary fractalkine was higher in IgAN patients with recurrent episodes of gross 157 hematuria compared with other patients with microscopic or no hematuria (Cox et al. 2012). 158 Blocking the CX3CR1 with anti CX3CR1 antibody stops the migration of leukocytes into the 159 glomeruli and prevents crescent formation and ameliorate renal function (Feng et al. 1999), thus inhibiting deterioration of renal function to ESRD in IgAN. On the other hand, CCL4 is the chemokine with specificity for CCR5 receptors. The d32-CCR5 polymorphism played a significant role in the progression of primary IGAN, with the $\mathrm{nl} / \mathrm{nl}$ genotype being an independent protective factor for late progression towards end-stage renal disease (Berthoux et al. 2006). Upregulation of CCR5 is demonstrated in the kidney and its expression is related to the increase in proteinuria (Navarro-Gonzalez et al. 2011). Therefore, we believe that these chemokines and chemokine receptors may play an important role in the pathogenesis of IgAN and a large number of studies should be conducted to clear the mechanism in the future.

The PPI network shows that some hub genes have high interaction with other genes, comparatively. High interaction of these genes demonstrates their potential participation in the manifestation and progression of IgAN. ITGB2 gene is the most interactive hub gene in the

172 network, and it is up-regulated, enriched in an inflammatory response and cell adhesion. ITGB2

173 gene is on chromosome 21 (21q22.3) and encodes integrin $\beta 2$ protein (CD11b/CD18) (Yassaee et 174 al. 2016). Upon inflammatory stimuli, CD11b/CD18 is rapidly activated via a conformational 
175

176

177 178

179

180

181

182

183

184

185

186

187

188

189

190

191

192

193

194

195

196

197

198

199

switch to mediate leukocyte migration from circulation to the inflamed tissue by binding to ICAM1(Hynes 2002). Recent studies revealed that inhibition of the CD11b/CD18 could prevent the acute kidney injury and the progression of acute kidney injury to chronic kidney disease (Dehnadi et al. 2017; Yago et al. 2015). Of note, the infiltration of the inflammatory cell is an important characteristic in IgAN (Wyatt \& Julian 2013). Therefore, inflammatory response and cell adhesion via CD11b/CD18 may have a significant effect in $\operatorname{IgAN}$ pathogenesis initiation.

The observation of the ALB gene in the PPI network is peculiar. The ALB is encoding albumin which is chiefly found in urinary protein. The ALB possesses high connectivity in the PPI network despite the gene being down-regulated with no significant pathway. These observations have resulted from the fact that kidney tubular epithelial cells are pathologically exposed to massive urinary proteins in patients with glomerular diseases (Remuzzi \& Bertani 1998). The experimental evidence demonstrated that urinary proteins, which include albumin, are involved in the mechanism of tubulointerstitial fibrosis (Eddy 2004; Remuzzi \& Bertani 1998). The ALB gene was down-regulated because in this bioinformatic analysis we use only the glomeruli tissues.

Although bioinformatics technology is a great method to identify the candidate genes contributing to diseases, many limitations still remain in this study. First, microarray data was downloaded from the GEO database instead of that developed by our research group. Second, the number of datasets and the sample size used in the analysis are small. Besides, the clinical data of the patients are not available, thus some confounding factors like age, sex, and renal function were not controlled in the analysis. Despite these limitations, our findings still have important implications for the molecular mechanisms of $\operatorname{IgAN}$ and further research is required to validate the results obtained in our paper.

\section{CONCLUSION}

In conclusion, the network analysis identifies several primary genes for IgAN. Comparatively, FN1 and ALB are the most common genes among all the genes in the three profiles. In-depth 
200

201

202

203

204

205

206

207

208

209

210

211

212

213

214

215

216

217

218

219

220

221

222

223

224

225

226

227

228

229

230

231

232

233

234

235

236

functional studies on these common genes may improve our understanding of the pathological processes of IgAN. However, these findings require experimental confirmation for future use.

\section{REFERENCES}

Altunkova I, Minkova V, and Belovezhdov N. 1993. Role of fibronectin in immune glomerulonephritis. Nephron 63:438-444.

Barbour SJ, Cattran DC, Kim SJ, Levin A, Wald R, Hladunewich MA, and Reich HN. 2013. Individuals of Pacific Asian origin with IgA nephropathy have an increased risk of progression to end-stage renal disease. Kidney Int 84:1017-1024. 10.1038/ki.2013.210

Benjamini Y, and Hochberg Y. 1995. Controlling the false discovery rate: a practical and powerful approach to multiple testing. J Royal Statistical Soc 1:289-300.

Berthoux FC, Berthoux P, Mariat C, Thibaudin L, Afiani A, and Linossier MT. 2006. CC-chemokine receptor five gene polymorphism in primary IgA nephropathy: the 32 bp deletion allele is associated with late progression to end-stage renal failure with dialysis. Kidney Int 69:565-572. 10.1038/sj.ki.5000106

Brotchie H, and Wakefield D. 1990. <Fibronectin structure function and significance in wound healing. Australas J Dermatol 31:47-56.

Cox SN, Sallustio F, Serino G, Loverre A, Pesce F, Gigante M, Zaza G, Stifanelli PF, Ancona N, and Schena FP. 2012. Activated innate immunity and the involvement of CX3CR1-fractalkine in promoting hematuria in patients with IgA nephropathy. Kidney Int 82:548-560.

10.1038/ki.2012.147

Cox SN, Sallustio F, Serino G, Pontrelli P, Verrienti R, Pesce F, Torres DD, Ancona N, Stifanelli P, Zaza G, and Schena FP. 2010. Altered modulation of WNT-beta-catenin and PI3K/Akt pathways in IgA nephropathy. Kidney Int 78:396-407. 10.1038/ki.2010.138

Dehnadi A, Benedict Cosimi A, Neal Smith R, Li X, Alonso JL, Means TK, and Arnaout MA. 2017. Prophylactic orthosteric inhibition of leukocyte integrin CD11b/CD18 prevents long-term fibrotic kidney failure in cynomolgus monkeys. Nat Commun 8:13899. 10.1038/ncomms13899

Donadio JV, and Grande JP. 2002. IgA nephropathy. N Engl J Med 347:738-748.

Eddy AA. 2004. Proteinuria and interstitial injury. Nephrol Dial Transplant 19:277-281. $10.1093 / \mathrm{ndt} / \mathrm{gfg} 533$

Feng Y, Chen S, Garcia GE, Xia Y, Siana MA, Botti P, Wilson CB, Harrison JK, and Bacon KB. 1999. Prevention of crescentic glomerulonephritis by immunoneutralization of the fractalkine receptor CX3CR1. Kidney Int 56:612-620.

Hynes RO. 2002. Integrins: Bidirectional, Allosteric Signaling Machines. Cell 110:673-687.

Idasiak-Piechocka I, Oko A, Pawliczak E, Kaczmarek E, and Czekalski S. 2010. Elevated urinary fibronectin excretion predicts poor outcome in patients with primary chronic glomerulonephritis. Nephron Clin Pract 116:c47-52. 10.1159/000314550 
237

238

239

240

241

242

243

244

245

246

247

248

249

250

251

252

253

254

255

256

257

258

259

260

261

262

263

264

265

266

267

268

269

270

271

272

273

274

275

276

277

Kauffmann A, Gentleman R, and Huber W. 2009. arrayQualityMetrics--a bioconductor package for quality assessment of microarray data. Bioinformatics 25:415-416. 10.1093/bioinformatics/btn647

Kubosawa, H., and Kondo Y. 1998. Alterations in the distribution of plasma fibronectin and the ultrastructure of podocytes in the peripheral glomerular loops in nephrotic rats. Virchows Arch 433:449-455.

Liu P, Lassen E, Nair V, Berthier CC, Suguro M, Sihlbom C, Kretzler M, Betsholtz C, Haraldsson B, Ju W, Ebefors K, and Nystrom J. 2017. Transcriptomic and Proteomic Profiling Provides Insight into Mesangial Cell Function in IgA Nephropathy. J Am Soc Nephrol 28:2961-2972. 10.1681/ASN.2016101103

Navarro-Gonzalez JF, Mora-Fernandez C, Muros de Fuentes M, and Garcia-Perez J. 2011. Inflammatory molecules and pathways in the pathogenesis of diabetic nephropathy. Nat Rev Nephrol 7:327-340. 10.1038/nrneph.2011.51

Nishimura M, Umehara H, Nakayama T, Yoneda O, Hieshima K, Kakizaki M, Dohmae N, Yoshie O, and Imai T. 2002. Dual Functions of Fractalkine/CX3C Ligand 1 in Trafficking of Perforin//Granzyme Bh Cytotoxic Effector Lymphocytes That Are Defined by CX3CR1 Expression. J Immunol 168:6173-6180.

Park J, Song KH, and Ha H. 2012. Fractalkine increases mesangial cell proliferation through reactive oxygen species and mitogen-activated protein kinases. Transplant Proc 44:1026-1028. 10.1016/j.transproceed.2012.03.045

Remuzzi G, and Bertani T. 1998. Pathophysiology of progressive nephropathies. N Engl J Med 339:1448-1456.

Ritchie ME, Phipson B, Wu D, Hu Y, Law CW, Shi W, and Smyth GK. 2015. limma powers differential expression analyses for RNA-sequencing and microarray studies. Nucleic Acids Res 43:e47. 10.1093/nar/gkv007

Robert T, Berthelot L, Cambier A, Rondeau E, and Monteiro RC. 2015. Molecular Insights into the Pathogenesis of IgA Nephropathy. Trends Mol Med 21:762-775. 10.1016/j.molmed.2015.10.003

Shui HA, Ka SM, Lin JC, Lee JH, Jin JS, Lin YF, Sheu LF, and Chen A. 2006. Fibronectin in blood invokes the development of focal segmental glomerulosclerosis in mouse model. Nephrol Dial Transplant 21:1794-1802. 10.1093/ndt/gfl113

Smoot ME, Ono K, Ruscheinski J, Wang PL, and Ideker T. 2011. Cytoscape 2.8: new features for data integration and network visualization. Bioinformatics 27:431-432. 10.1093/bioinformatics/btq675

Suzuki H, Kiryluk K, Novak J, Moldoveanu Z, Herr AB, Renfrow MB, Wyatt RJ, Scolari F, Mestecky J, Gharavi AG, and Julian BA. 2011. The pathophysiology of IgA nephropathy. J Am Soc Nephrol 22:1795-1803. 10.1681/ASN.2011050464

Szklarczyk D, Franceschini A, Kuhn M, Simonovic M, Roth A, Minguez P, Doerks T, Stark M, Muller J, Bork P, Jensen LJ, and von Mering C. 2011. The STRING database in 2011: functional interaction networks of proteins, globally integrated and scored. Nucleic Acids Res 39:D561-568. 10.1093/nar/gkq973

Wang X, Kang DD, Shen K, Song C, Lu S, Chang LC, Liao SG, Huo Z, Tang S, Ding Y, Kaminski N, Sibille E, Lin Y, Li J, and Tseng GC. 2012. An R package suite for microarray meta-analysis in 
278

279

280

281

282

283

284

285

286

287

288

289

290

291

292 quality control, differentially expressed gene analysis and pathway enrichment detection.

Bioinformatics 28:2534-2536. 10.1093/bioinformatics/bts485

Wyatt RJ, and Julian BA. 2013. IgA nephropathy. N Engl J Med 368:2402-2414. 10.1056/NEJMra1206793

Yago T, Petrich BG, Zhang N, Liu Z, Shao B, Ginsberg MH, and McEver RP. 2015. Blocking neutrophil integrin activation prevents ischemia-reperfusion injury. $J$ Exp Med 212:1267-1281. 10.1084/jem.20142358

Yassaee VR, Hashemi-Gorji F, Boosaliki S, and Parvaneh N. 2016. Mutation spectra of the ITGB2 gene in Iranian families with leukocyte adhesion deficiency type 1. Hum Immunol 77:191-195. 10.1016/j.humimm.2015.11.019

Zhuang Q, Cheng K, and Ming Y. 2017. CX3CL1/CX3CR1 Axis, as the Therapeutic Potential in Renal Diseases: Friend or Foe? Curr Gene Ther 17:442-452. 10.2174/1566523218666180214092536 


\section{Table 1 (on next page)}

Characteristic of datasets included in the analysis 


\begin{tabular}{lllcc}
\hline Series Number & Tissue & Platform & IgA Nephropathy & Health control \\
\hline GSE37460 & glomeruli & GPL14663 & 9 & 17 \\
GSE93798 & glomeruli & GPL22945 & 20 & 22 \\
GSE104948 & glomeruli & GPL24120 & 27 & 3 \\
\hline
\end{tabular}

1 
Table 2 (on next page)

Differentially Expressed Genes Identified in at least two datasets. 


\begin{tabular}{|c|c|c|c|c|c|}
\hline & \multicolumn{3}{|c|}{$\log _{2} \mathrm{FC}$} & \multicolumn{2}{|c|}{ Meta-P-value } \\
\hline & GSE37460 & GSE93798 & GSE104948 & Fisher & $\mathrm{MaxP}$ \\
\hline FN1 & 2.32 & 3.58 & 2.23 & $1.0 \mathrm{E}-20$ & $8.9 \mathrm{E}-06$ \\
\hline HBA1 & 3.50 & - & 3.53 & $1.0 \mathrm{E}-20$ & $1.0 \mathrm{E}-20$ \\
\hline HBB & 3.32 & - & 3.34 & $1.0 \mathrm{E}-20$ & $1.1 \mathrm{E}-04$ \\
\hline FCN1 & 1.99 & - & 2.85 & $1.0 \mathrm{E}-20$ & $2.1 \mathrm{E}-01$ \\
\hline TYROBP & 1.81 & - & 2.95 & $1.0 \mathrm{E}-20$ & $1.1 \mathrm{E}-01$ \\
\hline TIE1 & 1.71 & - & 1.92 & $1.0 \mathrm{E}-20$ & $1.8 \mathrm{E}-01$ \\
\hline IGFBP5 & 1.71 & 3.92 & - & $5.9 \mathrm{E}-06$ & $2.4 \mathrm{E}-03$ \\
\hline HCK & 1.69 & - & 2.42 & $1.0 \mathrm{E}-20$ & $9.3 \mathrm{E}-01$ \\
\hline FCER1G & 1.68 & - & 2.87 & $1.0 \mathrm{E}-20$ & $1.0 \mathrm{E}-20$ \\
\hline PTPRC & 1.64 & - & 3.14 & $1.0 \mathrm{E}-20$ & $1.0 \mathrm{E}-20$ \\
\hline NETO2 & 1.61 & - & 2.08 & $1.0 \mathrm{E}-20$ & $9.6 \mathrm{E}-04$ \\
\hline LY96 & 1.56 & - & 2.48 & $1.0 \mathrm{E}-20$ & $7.6 \mathrm{E}-03$ \\
\hline CD14 & 1.55 & - & 1.90 & $1.0 \mathrm{E}-20$ & $1.0 \mathrm{E}-20$ \\
\hline CD53 & 1.55 & - & 2.67 & $1.0 \mathrm{E}-20$ & 1.7E-03 \\
\hline CCL4 & 1.50 & - & 2.00 & $7.4 \mathrm{E}-07$ & $3.1 \mathrm{E}-02$ \\
\hline ITGB2 & 1.50 & - & 2.59 & $1.0 \mathrm{E}-20$ & 1.9E-01 \\
\hline IFI30 & 1.49 & - & 1.74 & $1.0 \mathrm{E}-20$ & $1.0 \mathrm{E}-20$ \\
\hline COL15A1 & 1.48 & - & 1.77 & $1.0 \mathrm{E}-20$ & $1.0 \mathrm{E}-20$ \\
\hline COL1A2 & 1.44 & - & 2.77 & $2.2 \mathrm{E}-06$ & $3.1 \mathrm{E}-01$ \\
\hline CX3CR1 & 1.42 & - & 3.45 & $1.0 \mathrm{E}-20$ & $2.5 \mathrm{E}-01$ \\
\hline COL6A3 & 1.41 & - & 2.05 & $1.5 \mathrm{E}-06$ & $1.0 \mathrm{E}-20$ \\
\hline CD44 & 1.40 & - & 3.12 & $1.0 \mathrm{E}-20$ & $1.0 \mathrm{E}-20$ \\
\hline CTSS & 1.40 & - & 2.37 & $1.0 \mathrm{E}-20$ & $3.1 \mathrm{E}-01$ \\
\hline LYN & 1.39 & - & 2.07 & $1.0 \mathrm{E}-20$ & $3.0 \mathrm{E}-01$ \\
\hline TBX3 & 1.32 & - & 2.26 & $1.0 \mathrm{E}-20$ & $3.3 \mathrm{E}-03$ \\
\hline
\end{tabular}




\begin{tabular}{lccccc} 
POSTN & 1.32 & 3.47 & - & $7.4 \mathrm{E}-07$ & $8.8 \mathrm{E}-05$ \\
SAMSN1 & 1.31 & - & 1.75 & $7.4 \mathrm{E} 07$ & $1.0 \mathrm{E}-20$ \\
CYBB & 1.30 & - & 2.28 & $1.0 \mathrm{E}-20$ & $5.6 \mathrm{E}-02$ \\
ALB & -1.67 & -3.42 & -2.39 & $4.4 \mathrm{E}-06$ & $3.7 \mathrm{E}-05$ \\
FOSB & -2.77 & -6.09 & - & $1.0 \mathrm{E}-20$ & $3.2 \mathrm{E}-01$ \\
NR4A3 & -2.10 & -3.76 & - & $1.0 \mathrm{E}-20$ & $2.4 \mathrm{E}-02$ \\
SIK1 & -2.07 & -3.42 & - & $1.0 \mathrm{E}-20$ & $3.2 \mathrm{E}-02$ \\
NR4A2 & -1.99 & -3.71 & - & $1.0 \mathrm{E}-20$ & $1.3 \mathrm{E}-03$ \\
ATF3 & -1.98 & -4.82 & - & $1.0 \mathrm{E}-20$ & $7.5 \mathrm{E}-01$ \\
MAFF & -1.71 & -3.62 & - & $6.0 \mathrm{E}-05$ & $8.4 \mathrm{E}-02$ \\
IGF1 & -1.60 & - & -1.82 & $1.0 \mathrm{E}-20$ & $1.0 \mathrm{E}-20$ \\
EGR1 & -1.44 & -4.19 & - & $5.6 \mathrm{E}-05$ & $9.4 \mathrm{E}-01$ \\
EGR3 & -1.29 & -3.32 & - & $5.0 \mathrm{E}-05$ & $8.9 \mathrm{E}-06$ \\
\hline
\end{tabular}

1 


\section{Table 3 (on next page)}

Pathways significantly enriched by up-regulated differentially expressed genes. 


\begin{tabular}{|c|c|c|c|c|}
\hline ID & Term & Count & $\mathrm{P}$ value & Genes \\
\hline hsa04064 & $\begin{array}{l}\text { NF-kappa B signaling } \\
\text { pathway }\end{array}$ & 4 & 0.002 & LYN, LY96, CCL4, CD14 \\
\hline hsa04512 & $\begin{array}{l}\text { ECM-receptor } \\
\text { interaction }\end{array}$ & 4 & 0.002 & CD44, COL6A3, COL1A2, FN1 \\
\hline hsa05146 & Amoebiasis & 4 & 0.003 & COL1A2, ITGB2, CD14, FN1 \\
\hline hsa05144 & Malaria & 3 & 0.009 & ITGB2, HBA1, HBB \\
\hline hsa05152 & Tuberculosis & 4 & 0.014 & FCER1G, ITGB2, CTSS, CD14 \\
\hline hsa04062 & $\begin{array}{l}\text { Chemokine signaling } \\
\text { pathway }\end{array}$ & 4 & 0.016 & LYN, HCK, CX3CR1, CCL4 \\
\hline hsa05133 & Pertussis & 3 & 0.020 & LY96, ITGB2, CD14 \\
\hline hsa04666 & $\begin{array}{l}\text { Fc gamma R-mediated } \\
\text { phagocytosis }\end{array}$ & 3 & 0.024 & PTPRC, LYN, HCK \\
\hline hsa04974 & $\begin{array}{l}\text { Protein digestion and } \\
\text { absorption }\end{array}$ & 3 & 0.026 & COL6A3, COL1A2, COL15A1 \\
\hline hsa04620 & $\begin{array}{l}\text { Toll-like receptor } \\
\text { signaling pathway }\end{array}$ & 3 & 0.037 & LY96, CCL4, CD14 \\
\hline hsa04650 & $\begin{array}{l}\text { Natural killer cell } \\
\text { mediated cytotoxicity }\end{array}$ & 3 & 0.048 & FCER1G, ITGB2, TYROBP \\
\hline hsa04611 & Platelet activation & 3 & 0.054 & LYN, COL1A2, FCER1G \\
\hline hsa04145 & Phagosome & 3 & 0.069 & ITGB2, CTSS, CD14 \\
\hline hsa05143 & $\begin{array}{l}\text { African } \\
\text { trypanosomiasis }\end{array}$ & 2 & 0.092 & HBA1, HBB \\
\hline
\end{tabular}

1 


\section{Figure 1}

A Venn diagram showing the differentially expressed genes in GSE37460, GSE93978, and GSE104948. (A) Up-regulated genes. (B) Down-regulated genes.

A

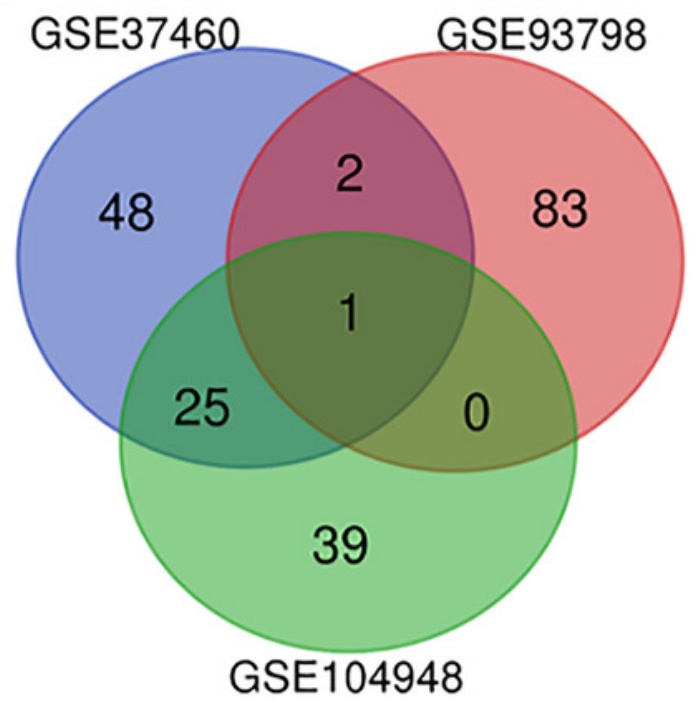

B

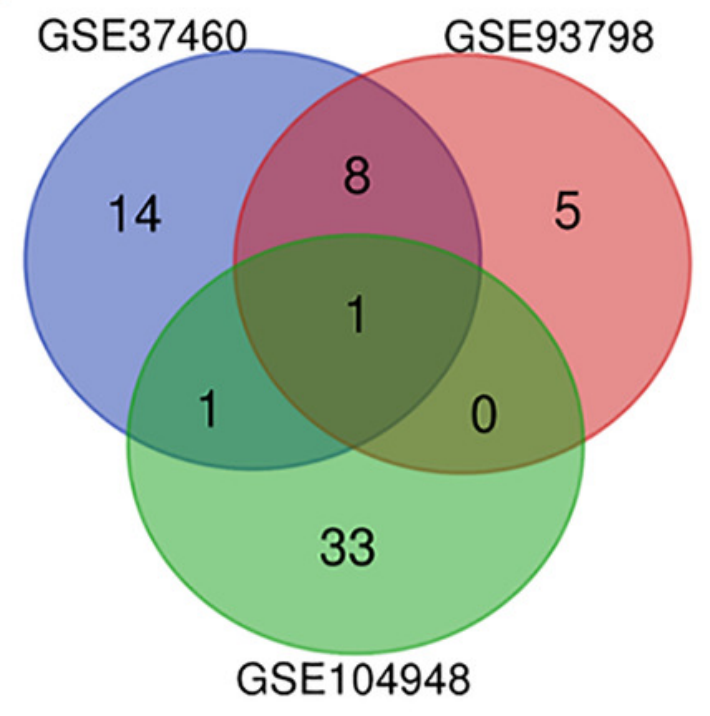


Figure 2 (on next page)

GO term of overlapped differentially expressed genes. Blue bars, biological process; green bars, cellular component; red bars, molecular function. (A) Up-regulated genes. (B) Down-regulated genes. 
A

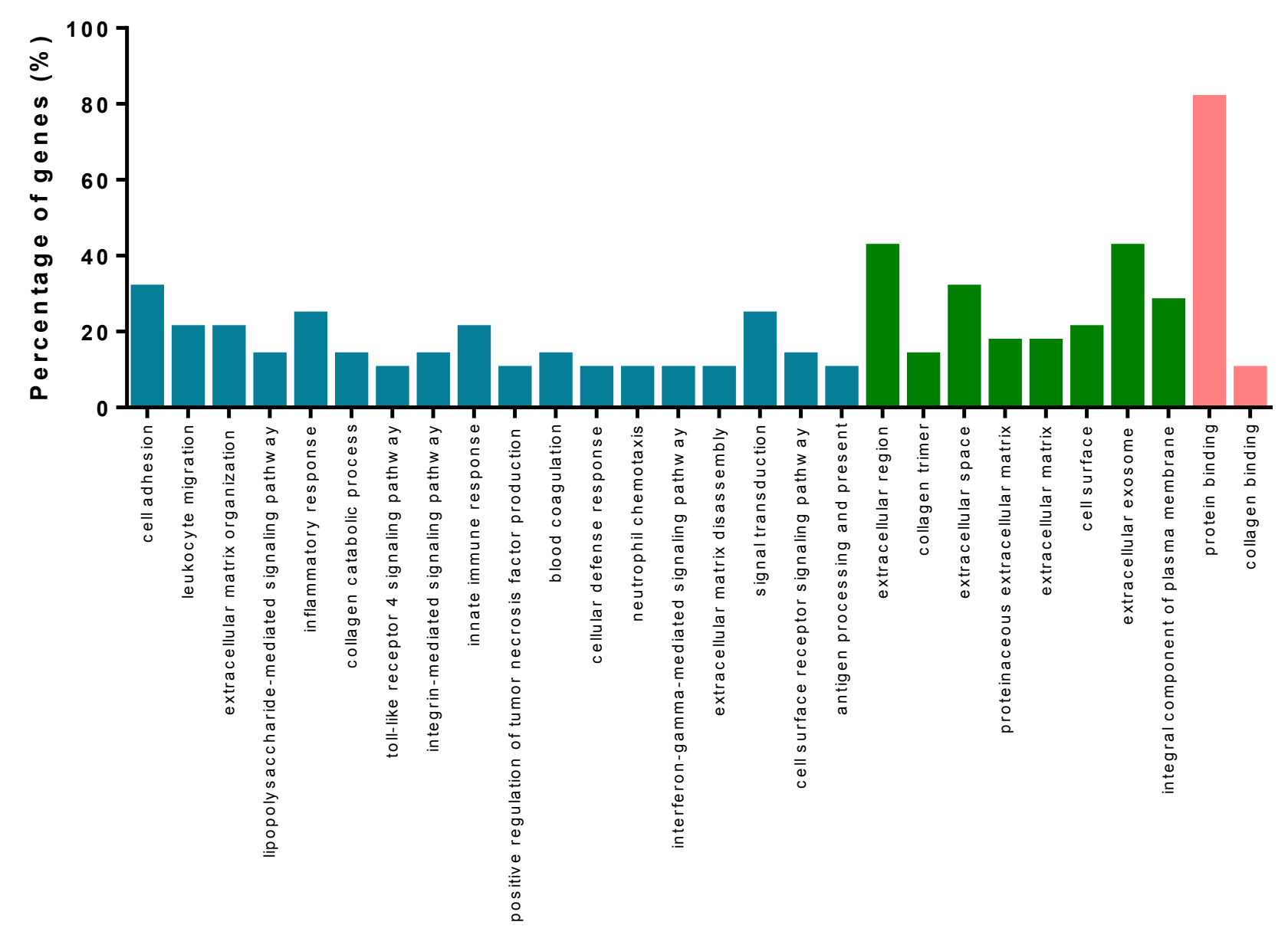

B

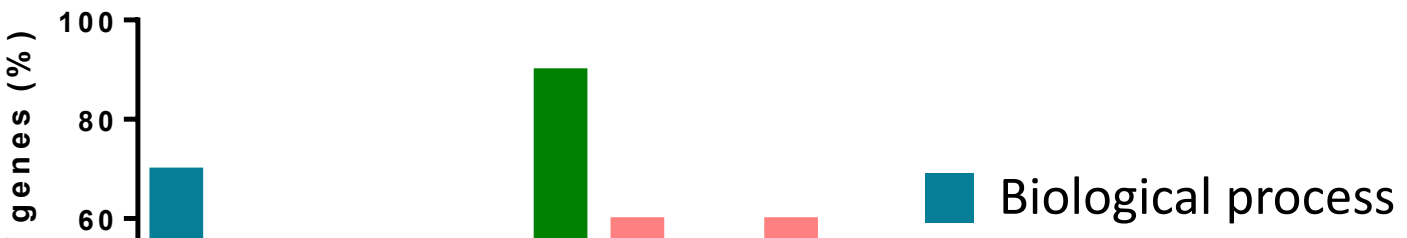

Cellular component Molecular function 
Figure 3

Flow chart of data selection and processing

Protein-protein interaction network of overlapped differentially expressed genes. Red circles, up-regulated genes; green circles, down-regulated genes

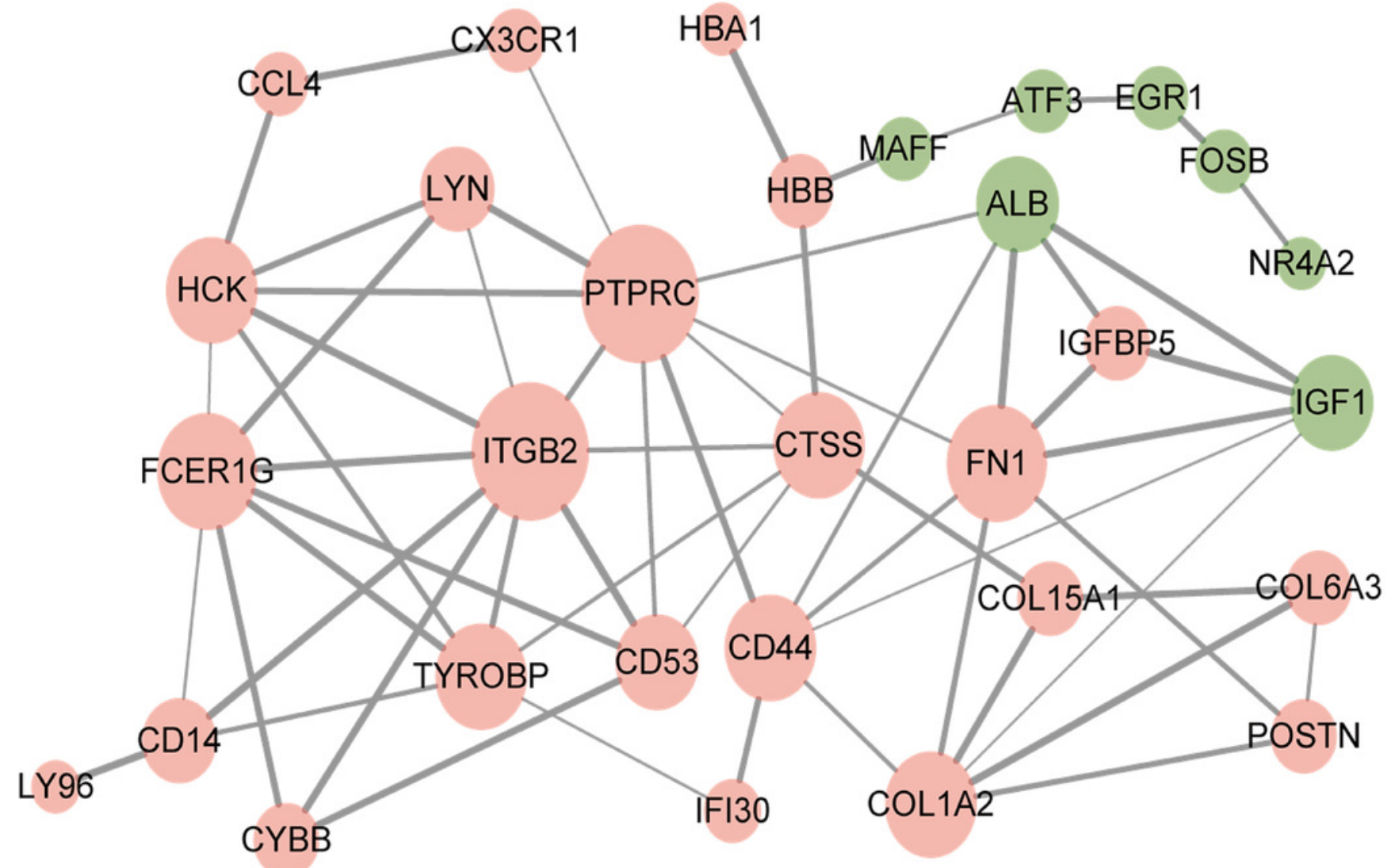

